Akute und chronische Rhinosinusitis

\title{
Wirkmechanismus von pflanzlichem Sekretolytikum aufgeklärt
}

- Bei Entzündungen der Nasennebenhöhlen ist die mukoziliäre Clearance gestört, zähflüssiges Sekret wird nicht mehr abtransportiert. Mit dem pflanzlichen Kombinationspräparat Sinupret ${ }^{\circledR}$ kann die Sekretolyse wieder in Gang gebracht werden. Wie das funktioniert, konnte jetzt durch In-vitro- und In-vivo-Untersuchungen aufgeklärt werden.

Die Störung der mukoziliären Clearance beruht darauf, dass die das zilientragende Epithel bedeckende, dünnflüssige Sekretschicht (Solphase) austrocknet. Dadurch ist die Beweglichkeit der Zilien eingeschränkt, und das visköse Sekret (Gelphase), das der Solphase aufliegt, kann nicht mehr Richtung Pharynx abtransportiert werden. Genau in diesen Prozess greift einer neuen Studie zufolge Sinupret ${ }^{\circledR}$ ein.

\section{Sekretion von Chloridionen nimmt zu} Wie die Studie zeigen konnte, erhöhen die in dem Phytotherapeutikum enthaltenen Flavonoide die Permeabilität des

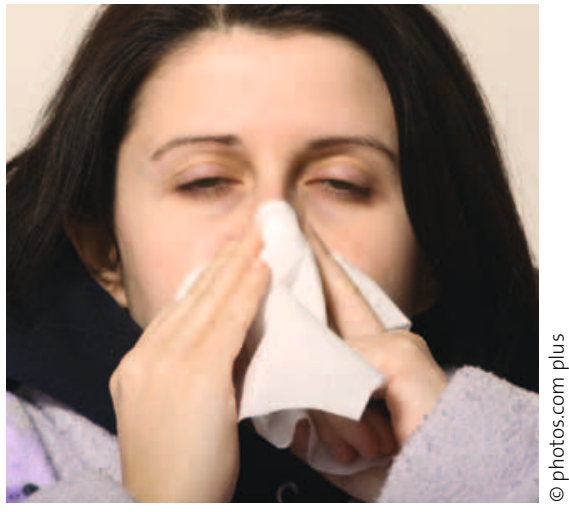

Durch Stimulierung des CFTR-Kanals steigert das Phytotherapeutikum die Sekretolyse.

transepithelialen Chloridkanals CFTR. Durch die gesteigerte Chloridionensekretion kommt es zu einer Rehydratation der Solphase und damit zur Wiederherstellung der Funktion des Flimmerepithels.

Untersucht wurde der Wirkmechanismus des Phytotherapeutikums an Naseneptihelzellen von Mäusen und bei Mäusen. In beiden Ansätzen bewirkten die Bioflavonoide im Vergleich zur Kontrolle einen hoch signifikanten Anstieg der transepithelialen Chloridionensekretion. Diese Zunahme fiel in vivo sogar stärker aus als mit Forskolin, einem der stärksten bekannten CFTR-Aktivatoren.

\section{Kombipräparat mit Mehrfachwirkung}

Bei dem Phytotherapeutikum Sinupret ${ }^{\circledR}$ handelt es sich um eine Fünffachkombination aus Enzianwurzel, Primelblüten, Gartenampferkraut, Holunderblüten und Eisenkraut. Das Kombinationspräparat wird zur Behandlung der akuten und chronischen Rhinosinusitis eingesetzt. Die Sekretolyse ist dabei nur eine der therapeutischen Wirkungen. Darüber hinaus sind für das Phytotherapeutikum auch antiphlogistische, antivirale, antibakterielle und immunmodulatorische Eigenschaften nachgewiesen.

- BS

Quelle: Virgin F et al. Laryngoscope 2010; 120(5):1051-56; Pressemitteilung Bionorica

\section{Neue Blutzuckermessgeräte für Alt und Jung}

- Mit zwei innovativen und modern gestalteten Blutzuckermessgeräten wird das Unternehmen Sanofi-Aventis voraussichtlich Anfang 2011 auf den Markt gehen. Die Geräte wurden erstmals beim europäischen Diabeteskongress in Stockholm vorgestellt.

Das größere BGStar ${ }^{\circledR}$ hat die Maße $83 \times 46 \times 20 \mathrm{~mm}$ und ist mit seinem übersichtlichen Display und den großen griffigen Tasten besonders leicht zu bedienen. Verschiedene Alarmfunktionen machen auf Abweichungen wie Hypo- und Hyperglykämien aufmerksam. Auch gibt es eine Rückmeldung über die Bedeutung von Ergebnissen und zur Glukosevariabilität. Ein positives Feedback kann auch in Form eines Smileys zugeschaltet werden.

Das mit $56 \times 24 \times 10 \mathrm{~mm}$ und nur 8,5 g deutlich kleinere iBGStar ${ }^{\circledR}$ funktioniert eigenständig und kann auch direkt an ein iPhone ${ }^{\circledR}$ oder einen iPodtouch ${ }^{\circledR}$ angedockt werden. Die
Messeinheit arbeitet in Verbindung mit dem iBGStar ${ }^{\circledR}$ Manager App der Apple-Geräte und erlaubt die detaillierte Auswertung und Darstellung der Messwerte.

Beide Geräte, die in Zusammenarbeit mit dem Unternehmen AgaMatrix entwickelt wurden, müssen nicht kodiert werden und kommen mit 0,5 $\mu$ l Blut für eine akkurate Messung von sechs Sekunden Dauer aus. Ein umfassendes Softwarepaket ist in der Entwicklung. - SPA

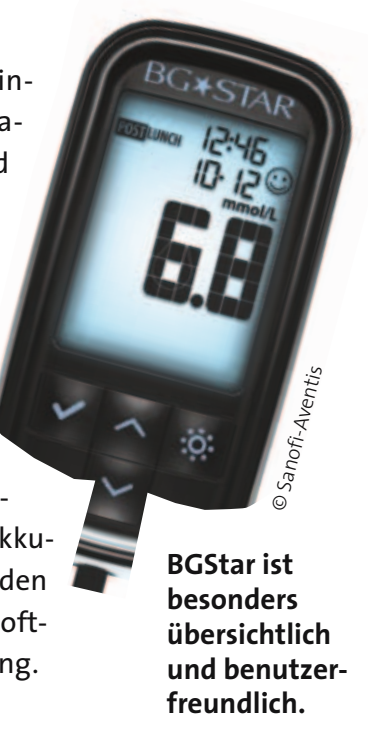

